\title{
Scleroderma Renal Crisis: A Pathology Perspective
}

\author{
Ibrahim Batal, ${ }^{1}$ Robyn T. Domsic, ${ }^{2}$ Thomas A. Medsger Jr., ${ }^{2}$ and Sheldon Bastacky ${ }^{1}$ \\ ${ }^{1}$ Department of Pathology, University of Pittsburgh Medical Center, A614 Scaife Hall, 200 Lothrop Street, Pittsburgh, PA 15213, USA \\ ${ }^{2}$ Division of Rheumatology and Clinical Immunology, University of Pittsburgh Medical Center, 3500 Terrace Street, \\ BST, S7, Pittsburgh, PA 15261, USA
}

Correspondence should be addressed to Ibrahim Batal, ibrahim_batal@yahoo.com

Received 14 May 2010; Accepted 28 June 2010

Academic Editor: Lorinda Chung

Copyright () 2010 Ibrahim Batal et al. This is an open access article distributed under the Creative Commons Attribution License, which permits unrestricted use, distribution, and reproduction in any medium, provided the original work is properly cited.

\begin{abstract}
Scleroderma renal crisis (SRC) is an infrequent but serious complication of systemic sclerosis (SSc). It is associated with increased vascular permeability, activation of coagulation cascade, and renin secretion, which may lead to the acute renal failure typically associated with accelerated hypertension. The histologic picture of SRC is that of a thrombotic microangiopathy process with prominent small vessel involvement manifesting as myxoid intimal changes, thrombi, onion skin lesions, and/or fibrointimal sclerosis. Renal biopsies play an important role in confirming the clinical diagnosis, excluding overlapping/superimposed diseases that might lead to acute renal failure in SSc patients, helping to predict the clinical outcome and optimizing patient management. Kidney transplantation may be the only treatment option available for a subset of SRC patients who develop end-stage renal failure despite aggressive angiotensin-converting enzyme inhibitor therapy. However, the posttransplant outcome for SSc patients is currently suboptimal compared to the general renal transplant population.
\end{abstract}

\section{Introduction}

Systemic sclerosis (SSc) is a multisystem autoimmune disorder that can manifest as either the diffuse cutaneous (dc) or the limited cutaneous (lc) variant, distinguished by the degree and the extent of cutaneous sclerosis [1]. Scleroderma renal crisis (SRC) can complicate the course of up to $10 \%$ of patients with SSc. Although most frequently seen in dcSSc, SRC can occur in patients with lcSSc $[2,3]$ and rarely in patients with no significant dermal sclerosis termed systemic sclerosis sine scleroderma (ssSSc) [4]. The etiology of SCR remains incompletely understood, with most models of pathogenesis suggesting an initial trigger of vascular endothelial injury. Alteration in cellular and/or humoral immunity may also play a role in SRC pathogenesis $[1,5,6]$. SSc has been associated with $\mathrm{T}$ helper lymphocyte type-2 (TH-2) activation, cytokine production (particularly Il-4, IL-13, and IL-17), and excess collagen accumulation, which could participate in the development of vasculopathy [7]. B cell activation has also been described in SSc patients [7]. The association between the presence of several specific autoantibodies and the development of SRC raises a potential contributing role of autoantibodies in the pathogenesis of SRC $[8,9]$. In addition, antiendothelial cell antibodies, which are capable of inducing endothelial cell apoptosis [10] have been detected in up to $85 \%$ of SSc patients [11]. Overexpression of endothelin-1, a protein that plays a role in blood vessel constriction, and its receptor endothelin-B has been demonstrated in the small vessels of two SRC patients [12]. Furthermore, the C4d complement degradation product product is regarded as an immunologic marker of antibody-mediated rejection in renal allografts, has been detected in native renal biopsies from a subset of SRC patients [13].

Subsequent to the potential endothelial triggering injury, the proposed cascade of histologic alterations is initiated by rapid increase in endothelial permeability and intimal edema. This then places the subendothelial connective tissue in direct contact with circulating blood elements activating the coagulation cascade and vascular thrombosis. The underlying connective tissue reacts to this insult by promoting fibroblastic and nonfibroblastic stromal proliferation, which manifests as proliferative endarteropathy (onion skin type lesion). Decreased renal perfusion as a result of arterial narrowing can additionally lead to juxtaglomerular apparatus (JGA) hyperplasia and renin secretion, resulting 
in accelerated hypertension and progressive renal injury. A milder form of vascular pathology, manifested usually as fibrointimal thickening can often be observed in SSc patients without SRC [14].

Adequate renal biopsy specimens are generally capable of reflecting the aforementioned pathophysiologic changes. A detailed histologic assessment can confirm the clinical diagnosis and help exclude potential overlapping or superimposed etiologies.

\section{Clinical and Laboratory Features}

SRC is typically characterized by a sudden and marked increase in systemic blood pressure (although normotensive SRC has been described [15]), and acute renal failure, with or without significant microangiopathic hemolytic anemia or thrombocytopenia. SRC is often accompanied by headache, blurring of vision, and dyspnea. These symptoms can be attributed to hypertensive encephalopathy, congestive heart failure, and/or pulmonary edema, respectively, as consequences of the rapid increase in blood pressure [16, 17]. Since SRC can present as acute renal failure, one would expect a significant elevation of serum creatinine and a considerable fall of glomerular filtration rate (GFR). In a relatively large study, the median serum creatinine value in SRC patients at presentation was $200 \mathrm{mmol} / \mathrm{l}(2.3 \mathrm{mg} / \mathrm{dl})$ [18]. In our study, the median serum creatinine value at the time of biopsy was $362 \mathrm{mmol} / \mathrm{l}(4.1 \mathrm{mg} / \mathrm{dl})$ [13].

SRC more frequently affects females than males [1]. This may reflect the overall increased prevalence of SSc in the female population. Rapid progression of skin thickening in patients with SSc [19] and high doses of corticosteroid therapy [20] are risk factors for the development of SRC. The latter is usually associated with systemic steroid administration [20]. However, rare cases of SRC have been described following topical steroid use [21]. In patients with SRC, presenting normal or mildly elevated blood pressures (normotensive SRC), older age and male sex have been suggested to be adverse prognostic factors [18]. The poor prognosis in normotensive SRC patients might reflect ongoing subclinical renal injury leading to severe irreversible destruction of renal parenchyma due to delayed diagnosis.

Virtually all SRC patients have detectable antinuclear antibodies (ANA). Anti-RNA polymerase antibodies (especially types I and III), which are the most frequent autoantibodies encountered in North American dcSSc patients, are significantly associated with the development of SRC $[8,9,22]$. An association with antitopoisomerase I (anti$\mathrm{Scl} 70$ ) antibodies in dcSSc patients has also been reported. In contrast, anticentromere antibodies, which are commonly detected in lcSSc, are rarely encountered [19]. Anti-U3 RNP antibodies were found to have an association with SRC in some [23] but not all [24] studies.

Microangiopathic hemolytic anemia occurs in up to half of SRC patients [25] and is characterized by abrupt onset of anemia, the presence of schistocytes in the peripheral blood smear, and thrombocytopenia. Thrombotic microan-

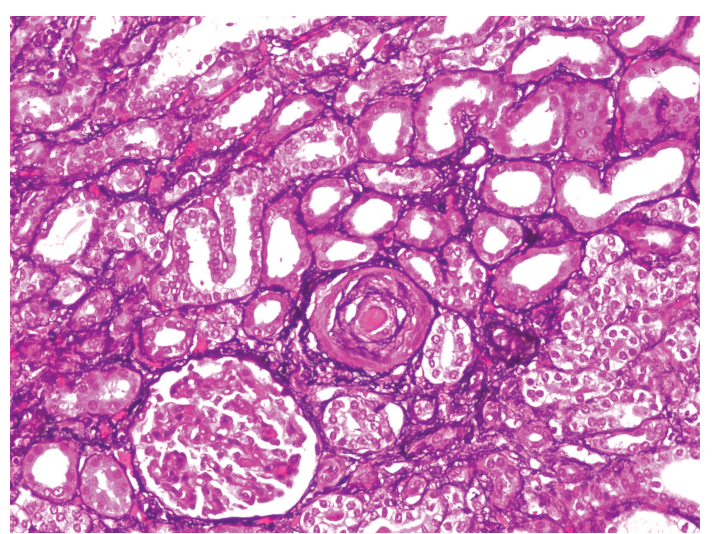

FIGURE 1: Arterial thrombosis associated with prominent glomerular ischemic collapse in a patient with scleroderma renal crisis (Methenamine silver stain; original magnification x100).

giopathy is often accompanied by elevated serum LDH and decreased haptoglobin.

\section{Gross Pathology}

Multiple, small petechial hemorrhages are frequently present on the surface of the affected kidneys. The cut section may reveal tiny wedge shaped infarcts and foci of cortical necrosis [26]. These changes are nonspecific and can be observed in other thrombotic microangiopathic disorders, such as hemolytic uremic syndrome, thrombotic thrombocytopenic purpura, and idiopathic malignant hypertension, or in association with some medications.

\section{Microscopic Pathology}

Renal biopsies, even though necessary to confirm the diagnosis, are not routinely warranted in SRC. Theoretically, unless the patient is suffering from typical clinical features and is associated with thrombotic microangiopathy picture on peripheral blood examination, the diagnosis cannot be confirmed with certainty without a renal biopsy. However, renal biopsy is an invasive procedure. Practically, such biopsies are recommended when doubt exists about the etiology of renal dysfunction, or, alternatively, to exclude the presence of other pathologic conditions.

The overall microscopic picture is that of a thrombotic microangiopathic process $[26,27]$. Similar to idiopathic malignant hypertension, and in contrast to hemolytic uremic syndrome and thrombotic thrombocytopenic purpura, primary small vessel changes usually predominate over glomerular alterations in SRC. Small vessel thrombi outnumbered glomerular thrombi in SRC [11/17 (65\%) versus $3 / 17(18 \%), P=.01$ ] [13], while the opposite was found in hemolytic uremic syndrome; thrombotic microangiopathy changes were more commonly detected in the glomeruli compared to small vessels [11/12 (92\%) versus 4/12 (33\%), $P=.009]$ [28]. 


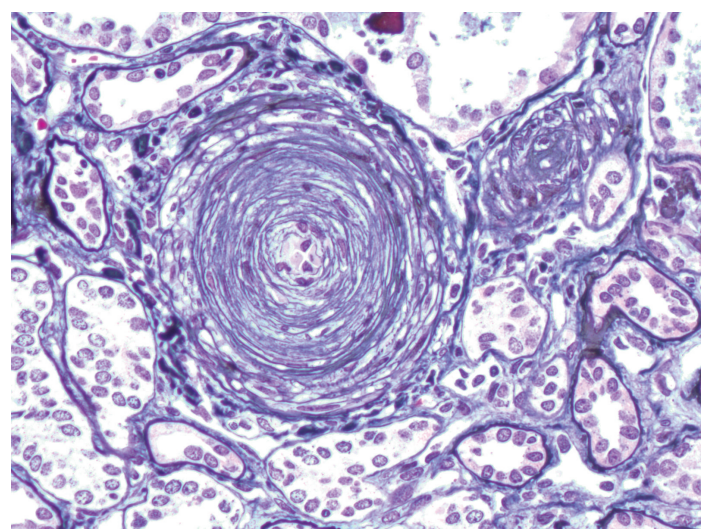

Figure 2: Prominent arterial onion skin lesion in a patient with scleroderma renal crisis. Such lesions often cause severe vascular narrowing leaving only a pinpoint open lumen (Methenamine silver stain; original magnification $\mathrm{x} 400$ ).

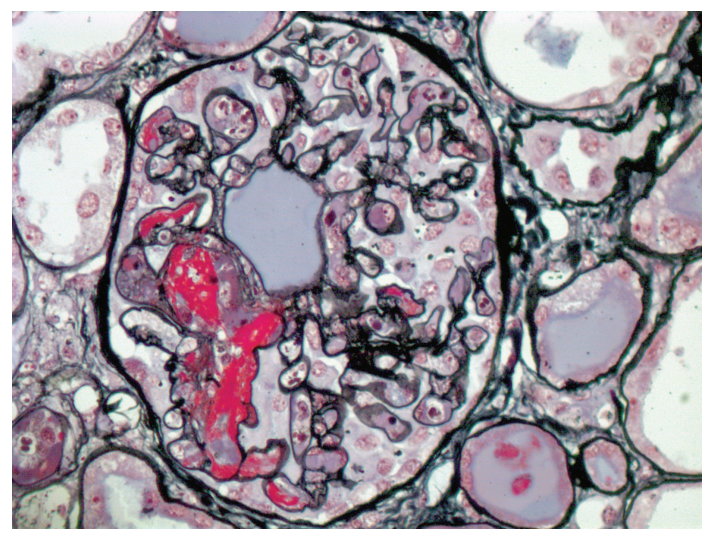

FIGURE 3: Glomerular capillary thrombosis in a patient with scleroderma renal crisis. This finding is rather infrequent in scleroderma renal crisis and is more commonly observed in hemolytic uremic anemia and thrombotic thrombocytopenic purpura (Methenamine silver stain; original magnification x600).

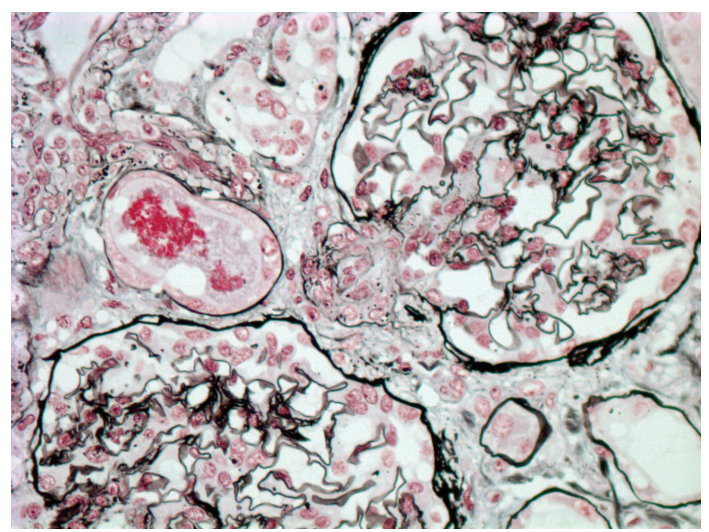

Figure 4: Prominent juxtaglomerular apparatus containing sparse silver positive renin granules in a patient with scleroderma renal crisis (Methenamine silver stain; original magnification $\mathrm{x} 400$ ).

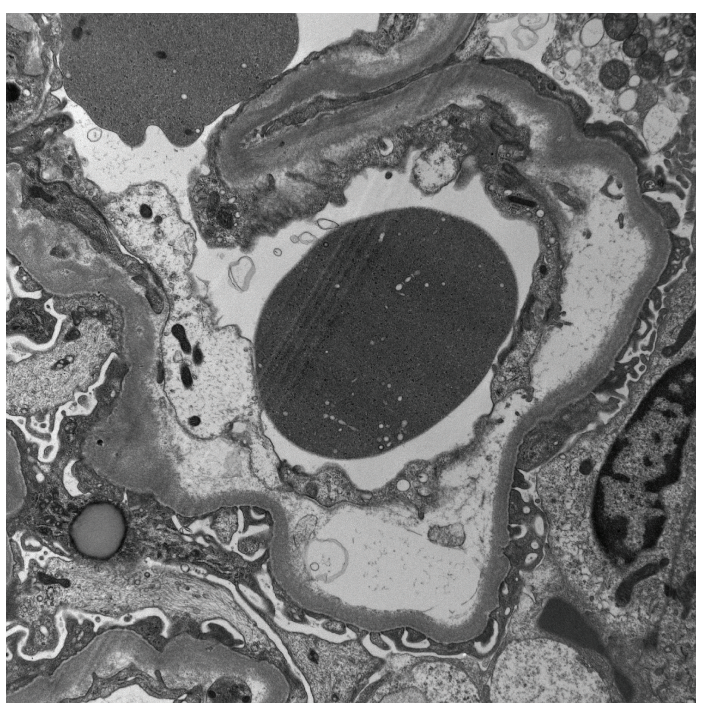

FIGURE 5: Electron microscopy from a patient with scleroderma renal crisis reveals detachment of the endothelium and prominent electron lucent fluffy material (Electron microscopy; original magnification $\mathrm{x} 5600$ ).

Histologic manifestations may vary during the course of the disease. Early vascular changes can manifest as intimal accumulation of myxoid material, thrombosis (Figure 1), and/or fibrinoid necrosis. Onion-skin lesions develop later (Figure 2), while fibrointimal sclerosis with or without adventitial fibrosis may be the only manifestation of chronic ongoing damage or organization resulting from previous episodes of acute injury. Acute glomerular changes can occur primarily or often develop secondary to the vascular injury and reduction in renal perfusion. Primary glomerular changes appear to be related to glomerular endothelial injury. These can manifest as endothelial swelling and glomerular capillary thrombosis (Figure 3). The latter is relatively infrequent [13]. Chronic glomerular changes, which include basement membrane double contours (tram tracking) and glomerulosclerosis, may develop later. Secondary glomerular changes may result in ischemic glomerular collapse. JGA hyperplasia, a histologic sequel of increased renin production can be observed microscopically (Figure 4). Prominent JGA hyperplasia was found to be present in $2 / 17$ (12\%) of our SRC cases [13]. Tubulointerstitial changes, which are also secondary to vascular pathology, are frequently manifested as ischemic acute tubular injury/necrosis or, if more chronic, as tubular atrophy and interstitial fibrosis. A lymphohistiocytic interstitial inflammatory infiltrate can occasionally be observed.

Finally, even though SRC represents an acute form of renal involvement, vascular pathology may be observed in SSc patients in the absence of SRC. Trostle et al. [14], in a case control autopsy study, compared the intimal surface areas of renal arteries in SSc autopsy cases (SRC, dcSSc without SRC, and lcSSc without SRC) to age- and sex-matched autopsy controls. Using morphometric techniques, these investigators confirmed that SRC patients had a significant 
increase in renal arterial intimal thickening. Interestingly, they also found that, in the absence of SRC, a significant increase in arterial fibrointimal thickness was observed in dcSSc patients, and to a lesser extent in lcSSc patients, compared to controls. Such vascular changes may be due to the presence of mild ongoing renal vascular injury below the threshold which triggers SRC.

\section{Ancillary Studies}

Immunofluorescence and electron microscopy are routinely used ancillary studies for evaluating native renal biopsies. Immunofluorescence studies are mainly utilized to characterize the presence, nature, pattern of staining, and anatomic distribution of immune deposits. Electron microscopy is used for ultrastructural assessment of renal biopsies. It is extremely helpful in localizing and characterizing immune complex and protein deposits with organized substructures (amyloidosis, fibrillary etc.) and to assess the glomerular endothelium, basement membrane, and podocytes.

Routine ancillary studies are of limited value in confirming the diagnosis of SRC. There are only a few reports which have characterized the immunofluorescence findings in SRC. Immunoglobulin deposits in the glomeruli and/or blood vessels were identified in most but not all of these studies. Among the immunoglobulin deposits, IgM, which might be considered the result of a nonspecific entrapment, was the most frequently detected [29-31]. This was often accompanied by complement deposits.

In SRC, electron microscopic evaluation frequently fails to detect discrete electron dense deposits. Hyaline material often accumulates in the subendothelium of the glomeruli and/or blood vessels in SRC [29-32]. Of note, hyaline deposits can sometimes be difficult to distinguish from definite immune complex deposits. Evidence of endothelial injury such as endothelial swelling and prominent accumulation of glomerular subendothelial electron lucent material have been described in SRC (Figure 5) but also in malignant hypertension [33]. Myointimal cells were detected in the extended fibrointima in SRC patients [34].

C4d is a complement split product which is generated following complement activation via classical or mannose-bound lectin pathways. C4d can be detected using immunoperoxidase on formalin fixed tissue or immunofluorescence on frozen tissue. The former is technically easier to perform while the latter is considered slightly more sensitive and specific. We identified finely granular C4d staining in the peritubular capillary of a subset of SRC patients who had associated poor renal outcome [13]. In allograft kidneys, the detection of peritubular capillary C4d staining is usually associated with antibody-mediated rejection and poor allograft outcome [35-37]. Confocal immunofluorescence can potentially play an important role in localizing $\mathrm{C} 4 \mathrm{~d}$ as a target. Although a larger multicenter study using immunofluorescence technique is needed to validate our preliminary findings and to further characterize the cause of such deposits, evidence supporting the role of antibodymediated injury in SSc/SRC patients is accumulating. First,

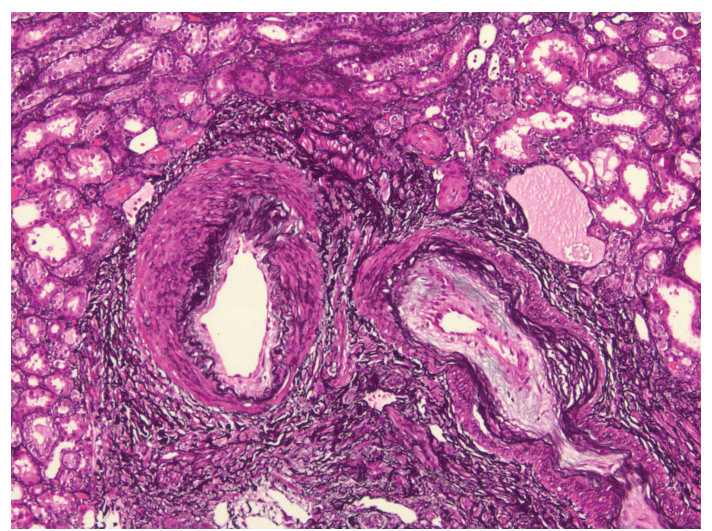

Figure 6: Prominent arterial adventitial fibrosis in a patient with scleroderma renal crisis. Note that the arteries also have mild intimal accumulation of myxoid material. (Methenamine silver stain; original magnification $\mathrm{x} 100)$.

disease-specific serum autoantibodies have been found to be associated with certain clinical manifestations [22]. Second, antiendothelial antibodies and increased expression of endothelin-1/endothelin-B have been detected in a subset of SRC patients [12]; it is noteworthy that overexpression of endothelin-1 gene has been recently discovered in allograft kidneys with antibody-mediated rejection [38]. Lastly, antiglobulin antibodies have been found in the eluate of several SRC kidney samples [30].

\section{Differential Diagnosis}

Clinically, SRC should be suspected when acute renal failure (ARF) develops in SSc patients. Nevertheless, ARF occurring in SSc patients is not always due to SRC. Renal artery stenosis, hypovolemia, crescentic glomerulonephritis (GN), and other renal diseases may also occur in SSc patients [27]. These disorders may result in a similar clinical picture. A thrombotic microangiopathy-like clinical picture can even be encountered in patients with renal arterial stenosis [27]. Distinguish SRC from crescentic GN is critical since immunosuppressive therapy is used to treat the latter. As the name implies, the presence of crescents is the hallmark of crescentic GN. In typical SRC cases, crescents are extremely rare and, when detected, are very small [39]. In SSc patients, most of the encountered crescentic GN are ANCA-associated. These are pauci-immune on immunofluorescence studies, associated with anti-myeloperoxidase antibodies, and usually triggered by penicillamine $[40,41]$. Less often, one may encounter immune complex GN [39] or antiglomerular basement membrane GN [42]. Immunofluorescence studies reveal granular glomerular basement membrane and/or mesangial immune complex deposits in the former and linear glomerular basement membrane IgG staining in the latter.

Histologically, thrombotic microangiopathic changes can be observed in several disorders. Although it is often impossible to ascertain the specific cause of thrombotic microangiopathy based on histologic evaluation alone, it 


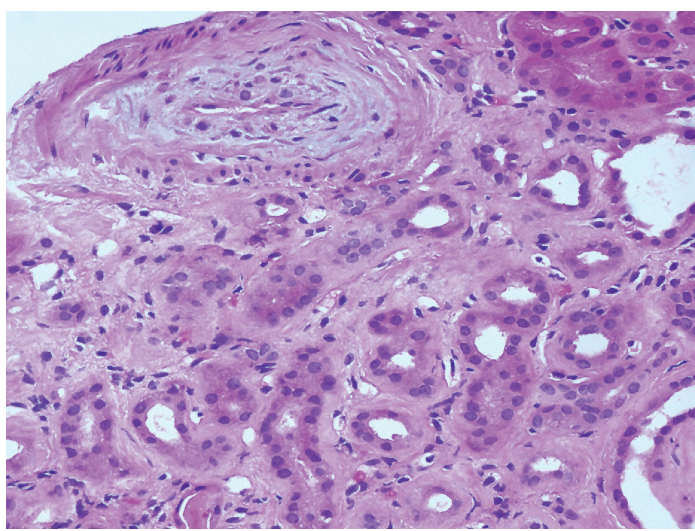

(a)

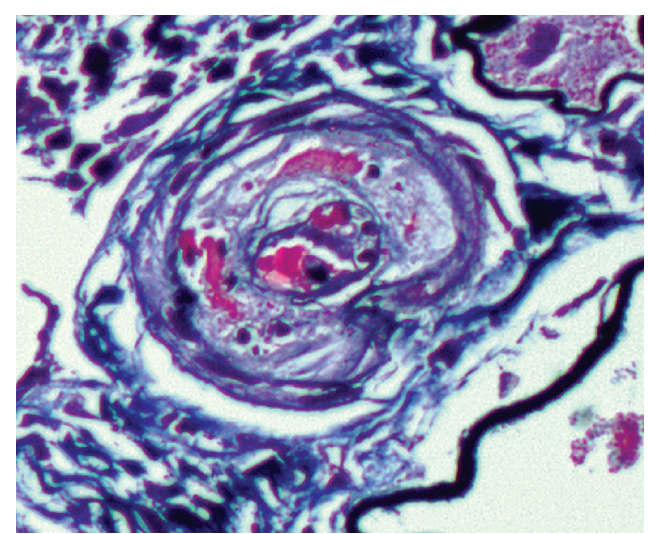

(b)

FIgURE 7: Two renal allograft biopsies with histologic features suspicious for recurrence of scleroderma. Note the prominent myxoid changes in the artery in biopsy (a) as well as the severe intimal thickening of blood vessels, which is accompanied by thrombosis and schistocytes within the arteriole wall in biopsy (b). The differential diagnosis includes acute antibody-mediated rejection and acute calcineurin inhibitor toxicity. Clinical correlation with the presence of C4d stain, detection of circulating donor-specific antibodies, and calcineurin inhibitor levels are usually warranted to achieve a correct diagnosis [(a) H\&E; original magnification x200 and (b) Methenamine silver stain; original magnification $\mathrm{x} 400]$.

is important to note that extraglomerular small vessel vascular lesions often predominate in SRC while primary glomerular capillary microangiopathic changes (glomerular capillary thrombosis) are a relatively infrequent histologic finding in SRC [13]. The presence of JGA hyperplasia has been described in SRC patients $[43,44]$. Similarly, vascular adventitial fibrosis (Figure 6) [45] has been regarded by some investigators to be characteristic for SSc [46] and SRC [47]. Clinicopathological correlation, however, is often required to achieve the correct diagnosis. In advanced stages of SRC, the histologic findings are usually nonspecific and reflect advanced chronic renal damage. At such late stages, it is often difficult to distinguish chronic vascular changes associated with organized SRC from preexisting chronic accelerated essential hypertension. The presence of periadventitial fibrosis, if prominent, might be helpful as a point in favor of SRC.

\section{Prognosis}

Several retrospective studies have investigated the role of renal biopsy in predicting prognosis in SRC. Penn et al. showed that the presence of acute vascular changes (myxoid intimal thickening and thrombosis) were associated with poor prognosis [18]. We showed that the severity and extent of acute vascular injury, including fibrinoid changes and/or thrombosis, was most predictive of poor outcome [13]. We also showed that severe glomerular ischemic collapse, and to a lesser extent acute tubular necrosis, may also be associated with poor prognosis [13]. Both of the latter are secondary changes reflecting the severity of vascular lesions. In contrast to acute changes, we observed that chronic renal changes did not significantly correlate with poor outcome [13]. A recently published abstract suggested that chronic pathological changes might be associated with a favorable prognosis [48]. The latter observation is difficult to explain since chronic changes are typically irreversible and are expected, if any, to have an adverse impact on renal survival, as was described in other kidney diseases such as lupus nephritis [49].

\section{Treatment/Outcome}

Blood pressure should be vigorously and aggressively controlled in patients with established SRC. The mortality associated with SRC has significantly decreased due to early diagnosis and aggressive angiotensin-converting enzyme (ACE) inhibitor therapy $[50,51]$. Still, a subset of SRC patients may be refractory to ACE-inhibitor and other hypertensive therapy. These patients often remain on dialysis or die $[17,51,52]$. Kidney transplantation should be considered if the condition does not reverse despite aggressive treatment (usually within two years) [53].

\section{Posttransplant Outcome}

Although renal transplantation offers superior survival in SRC patients, graft survival is frequently reduced in SScinduced renal failure compared to the general renal transplant population $[54,55]$. We retrospectively studied the posttransplantation course of 10 SRC patients [56]. One, three, and five year graft survivals in this SRC cohort of patients were $70 \%, 70 \%$, and $25 \%$, respectively, compared with approximately 90\%, 79\%, and 75\% graft survival in miscellaneous patients who received kidney transplants from deceased donors at the same institution [57]. Recurrence of scleroderma (Figure 7) may play a role in this poor postrenal transplant outcome $[58,59]$. Two of our 10 patients had histologic features suspicious for SRC recurrence, manifested by both exacerbated development of arterial fibrointimal 
thickening with thrombotic microangiopathy-like changes [56]. Pham et al. [59] found that recurrent SRC occurs early in the course of transplantation (within 2 years posttransplantation). However, Cheung et al. [58] challenged the conventional experience by reporting a recurrence of SRC which occurred seven years post-transplantation. This SRC occurred following switching therapy from an ACE inhibitor to an angiotensin II receptor blocker.

Establishing a histologic diagnosis of recurrent scleroderma/SRC in an allograft is more challenging than diagnosing SRC in a native kidney biopsy. In addition to recurrent SRC, the pathologic differential diagnosis in allograft biopsies with a thrombotic microangiopathy-like picture also includes antibody-mediated rejection, calcineurin inhibitor toxicity, infection, and other less common allograft-related abnormalities [60]. More chronic changes can also be difficult to distinguish from de novo transplant glomerulopathy.

In summary, SRC is a severe complication of systemic sclerosis. Although not always clinically warranted, renal biopsy can play an important role in establishing the diagnosis and in excluding other pathologic conditions such as vasculitis and connective tissue disease related and nonrelated syndromes. Furthermore, renal biopsies can help to predict renal prognosis and may contribute to our better understanding of the mechanisms and pathologic manifestations of SRC, ultimately leading to optimization of treatment strategies.

\section{References}

[1] L. I. Sakkas, "New developments in the pathogenesis of systemic sclerosis," Autoimmunity, vol. 38, no. 2, pp. 113-116, 2005.

[2] T. Sugimoto, M. Sanada, and A. Kashiwagi, "Is scleroderma renal crisis with anti-centromere antibody-positive limited cutaneous systemic sclerosis overlooked in patients with hypertension and/or renal dysfunction?" Nephrology, vol. 13, no. 2, pp. 179-180, 2008.

[3] T. Sugimoto, M. Soumura, K. Danno et al., "Scleroderma renal crisis in a patient with anticentromere antibody-positive limited cutaneous systemic sclerosis," Modern Rheumatology, vol. 16, no. 5, pp. 309-311, 2006.

[4] E. A. Gonzalez, E. Schmulbach, and B. Bastani, "Scleroderma renal crisis with minimal skin involvement and no serologic evidence of systemic sclerosis," American Journal of Kidney Diseases, vol. 23, no. 2, pp. 317-319, 1994.

[5] C. Chizzolini, "Update on pathophysiology of scleroderma with special reference to immunoinflammatory events," Annals of Medicine, vol. 39, no. 1, pp. 42-53, 2007.

[6] S. Sato, M. Fujimoto, M. Hasegawa, K. Takehara, and T. F. Tedder, "Altered B lymphocyte function induces systemic autoimmunity in systemic sclerosis," Molecular Immunology, vol. 41, no. 12, pp. 1123-1133, 2004.

[7] L. I. Sakkas, I. C. Chikanza, and C. D. Platsoucas, "Mechanisms of disease: the role of immune cells in the pathogenesis of systemic sclerosis," Nature Clinical Practice Rheumatology, vol. 2, no. 12, pp. 679-685, 2006.

[8] M. Chang, R. J. Wang, D. T. Yangco, G. C. Sharp, G. R. Komatireddy, and R. W. Hoffman, "Analysis of autoantibodies against RNA polymerases using immunoaffinitypurifed RNA polymerase I, II, and III antigen in an enzyme-linked immunosorbent assay," Clinical Immunology and Immunopathology, vol. 89, no. 1, pp. 71-78, 1998.

[9] M. Santiago, M. Baron, M. Hudson, R. W. Burlingame, and M. J. Fritzler, "Antibodies to RNA polymerase III in systemic sclerosis detected by ELISA," Journal of Rheumatology, vol. 34, no. 7, pp. 1528-1534, 2007.

[10] M. Worda, R. Sgonc, H. Dietrich et al., "In vivo analysis of the apoptosis-inducing effect of anti-endothelial cell antibodies in systemic sclerosis by the chorionallantoic membrane assay," Arthritis and Rheumatism, vol. 48, no. 9, pp. 2605-2614, 2003.

[11] C. Mihai and J. W. C. Tervaert, "Anti-endothelial cell antibodies in systemic sclerosis," Annals of the Rheumatic Diseases, vol. 69, no. 2, pp. 319-324, 2010.

[12] H. Kobayashi, T. Nishimaki, S. Kaise et al., "Immunohistological study of endothelin-1 and endothelin-A and B receptors in two patients with scleroderma renal crisis," Clinical Rheumatology, vol. 18, no. 5, pp. 425-427, 1999.

[13] I. Batal, R. T. Domsic, A. Shafer et al., "Renal biopsy findings predicting outcome in scleroderma renal crisis," Human Pathology, vol. 40, no. 3, pp. 332-340, 2009.

[14] D. C. Trostle, C. D. Bedetti, V. D. Steen, M. R. Al-Sabbagh, B. Zee, and T. A. Medsger Jr., "Renal vascular histology and morphometry in systemic sclerosis. A case-control autopsy study," Arthritis and Rheumatism, vol. 31, no. 3, pp. 393-400, 1988.

[15] D. J. Helfrich, B. Banner, V. D. Steen, and T. A. Medsger Jr., "Normotensive renal failure in systemic sclerosis," Arthritis and Rheumatism, vol. 32, no. 9, pp. 1128-1134, 1989.

[16] Y. S. Haviv and R. Safadi, "Normotensive scleroderma renal crisis: case report and review of the literature," Renal Failure, vol. 20, no. 5, pp. 733-736, 1998.

[17] B. Lewandowski, I. Domysławska, P. A. Klimiuk, and S. Sierakowski, "Kidney crisis in systemic sclerosis," Roczniki Akademii Medycznej w Bialymstoku, vol. 50, pp. 294-296, 2005.

[18] H. Penn, A. J. Howie, E. J. Kingdon et al., "Scleroderma renal crisis: patient characteristics and long-term outcomes," QJM, vol. 100, no. 8, pp. 485-494, 2007.

[19] C. P. Denton, G. Lapadula, L. Mouthon, and U. MüllerLadner, "Renal complications and scleroderma renal crisis," Rheumatology, vol. 48, pp. iii32-35, 2009.

[20] V. D. Steen and T. A. Medsger Jr., "Case-control study of corticosteroids and other drugs that either precipitate or protect from the development of scleroderma renal crisis," Arthritis and Rheumatism, vol. 41, no. 9, pp. 1613-1619, 1998.

[21] H. Sarwar, L. R. Espinoza, H. McGrath Jr., Z. Yaqub, and E. Cucurull, "Scleroderma renal crisis following widespread application of topical triamcinolone," Journal of Clinical Rheumatology, vol. 10, no. 5, pp. 275-276, 2004.

[22] V. D. Steen, "Autoantibodies in systemic sclerosis," Seminars in Arthritis and Rheumatism, vol. 35, no. 1, pp. 35-42, 2005.

[23] F. C. Arnett, J. D. Reveille, R. Goldstein et al., "Autoantibodies to fibrillarin in systemic sclerosis (scleroderma): an immunogenetic, serologic, and clinical analysis," Arthritis and Rheumatism, vol. 39, no. 7, pp. 1151-1160, 1996.

[24] R. Aggarwal, M. Lucas, N. Fertig, C. V. Oddis, and T. A. Medsger, "Anti-U3 RNP autoantibodies in systemic sclerosis," Arthritis and Rheumatism, vol. 60, no. 4, pp. 1112-1118, 2009.

[25] V. D. Steen and T. A. Medsger Jr., "Long-term outcomes of scleroderma renal crisis," Annals of Internal Medicine, vol. 133, no. 8, pp. 600-603, 2000.

[26] E. R. Fisher and G. P. Rodnan, "Pathologic observations concerning the kidney in progressive systemic sclerosis," A. M. A. Archives of Pathology, vol. 65, no. 1, pp. 29-39, 1958. 
[27] L. Mouthon, A. Bérezné, G. Bussone, L.-H. Noël, P. M. Villiger, and L. Guillevin, "Scleroderma renal crisis: a rare but severe complication of systemic sclerosis," Clinical Reviews in Allergy and Immunology. In press.

[28] I. Tostivint, B. Mougenot, A. Flahault et al., "Adult haemolytic and uraemic syndrome: causes and prognostic factors in the last decade," Nephrology Dialysis Transplantation, vol. 17, no. 7, pp. 1228-1234, 2002.

[29] D. Lapenas, G. P. Rodnan, and T. Cavallo, "Immunopathology of the renal vascular lesion of progressive systemic sclerosis (scleroderma)," American Journal of Pathology, vol. 91, no. 2, pp. 243-258, 1978.

[30] R. C. McCoy, C. C. Tisher, P. F. Pepe, and L. A. Cleveland, "The kidney in progressive systemic sclerosis. Immunohistochemical and antibody elution studies," Laboratory Investigation, vol. 35, no. 2, pp. 124-131, 1976.

[31] A. R. McGiven, W. G. De Boer, and A. J. Barnett, "Renal immune deposits in scleroderma," Pathology, vol. 3, no. 2, pp. 145-150, 1971.

[32] F. G. Silva and C. L. Pirani, "Electron microscopic study of medical diseases of the kidney: update-1988," Modern Pathology, vol. 1, no. 4, pp. 292-315, 1988.

[33] R. A. Sinclair, T. T. Antonovych, and F. K. Mostofi, "Renal proliferative arteriopathies and associated glomerular changes. A light and electron microscopic study," Human Pathology, vol. 7, no. 5, pp. 565-588, 1976.

[34] M. I. Salomon, J. Lamovec, and V. Tcherkoff, "Renal lesions in scleroderma," Angiology, vol. 29, no. 8, pp. 569-578, 1978.

[35] R. B. Colvin, "Antibody-mediated renal allograft rejection: diagnosis and pathogenesis," Journal of the American Society of Nephrology, vol. 18, no. 4, pp. 1046-1056, 2007.

[36] H. E. Feucht, H. Schneeberger, G. Hillebrand et al., "Capillary deposition of C4d complement fragment and early renal graft loss," Kidney International, vol. 43, no. 6, pp. 1333-1338, 1993.

[37] I. Batal, A. Girnita, A. Zeevi et al., "Clinical significance of the distribution of C4d deposits in different anatomic compartments of the allograft kidney," Modern Pathology, vol. 21, no. 12, pp. 1490-1498, 2008.

[38] B. Sis and P. F. Halloran, "Endothelial transcripts uncover a previously unknown phenotype: C4d-negative antibodymediated rejection," Current Opinion in Organ Transplantation, vol. 15, no. 1, pp. 42-48, 2010.

[39] A. Ramaswami, T. Kandaswamy, T. Rajendran et al., "Scleroderma with crescentic glomerulonephritis: a case report," Journal of Medical Case Reports, vol. 2, article 151, 2008.

[40] H. Endo, T. Hosono, and H. Kondo, "Antineutrophil cytoplasmic autoantibodies in 6 patients with renal failure and systemic sclerosis," Journal of Rheumatology, vol. 21, no. 5, pp. 864-870, 1994.

[41] D. L. Kamen, F. M. Wigley, and A. N. Brown, "Antineutrophil cytoplasmic antibody-positive crescentic glomerulonephritis in scleroderma- a different kind of renal crisis," Journal of Rheumatology, vol. 33, no. 9, pp. 1886-1888, 2006.

[42] T. Namba, M. Hatanaka, A. Takahashi et al., "A case of scleroderma with rapid progressive glomerulonephritis associated with both MPO-ANCA and anti-GBM antibodies," Nippon Jinzo Gakkai Shi, vol. 50, no. 1, pp. 64-68, 2008.

[43] M. Kirschstein, U. Helmchen, R. Jensen, R. M. Küster, and H. Lehmann, "Kidney involvement in a 17 -year-old boy with eosinophilic fasciitis," Clinical Nephrology, vol. 52, no. 3, pp. 183-187, 1999.

[44] R. A. Stone, C. C. Tisher, H. K. Hawkins, and R. R. Robinson, "Juxtaglomerular hyperplasia and hyperreninemia in progressive systemic sclerosis complicated by acute renal failure," American Journal of Medicine, vol. 56, no. 1, pp. 119123, 1974.

[45] P. J. Cannon, M. Hassar, D. B. Case, W. J. Casarella, S. C. Sommers, and E. C. LeRoy, "The relationship of hypertension and renal failure in scleroderma (progressive systemic sclerosis) to structural and functional abnormalities of the renal cortical circulation," Medicine, vol. 53, no. 1, pp. 1-46, 1974.

[46] V. Steen, C. P. Denton, J. E. Pope, and M. Matucci-Cerinic, "Digital ulcers: overt vascular disease in systemic sclerosis," Rheumatology, vol. 48, pp. iii19-24, 2009.

[47] V. D. Steen, "Renal involvement in systemic sclerosis," Clinics in Dermatology, vol. 12, no. 2, pp. 253-258, 1994.

[48] H. Penn, A. J. Howie, R. J. Stratton, et al., "The prognostic value of renal biopsy in scleroderma renal crisis," Arhtritis and Rheumatism, vol. 56, pp. 53-54, 2007, abstract \#3.

[49] M. G. Hunter, S. Hurwitz, C. O. C. Bellamy, and J. S. Duffield, "Quantitative morphometry of lupus nephritis: the significance of collagen, tubular space, and inflammatory infiltrate," Kidney International, vol. 67, no. 1, pp. 94-102, 2005.

[50] D. A. Collins, S. Patel, J. B. Eastwood, and B. E. Bourke, "Favourable outcome of scleroderma renal crisis," Journal of the Royal Society of Medicine, vol. 89, no. 1, pp. 49-50, 1996.

[51] V. D. Steen, J. P. Costantino, A. P. Shapiro, and T. A. Medsger Jr., "Outcome of renal crisis in systemic sclerosis: relation to availability of angiotensin converting enzyme (ACE) inhibitors," Annals of Internal Medicine, vol. 113, no. 5, pp. 352-357, 1990.

[52] E. Y. Rhew and W. G. Barr, "Scleroderma renal crisis: new insights and developments," Current Rheumatology Reports, vol. 6, no. 2, pp. 129-136, 2004.

[53] E. C. LeRoy and R. M. Fleischmann, "The management of renal scleroderma. Experience with dialysis, nephrectomy and transplantation," American Journal of Medicine, vol. 64, no. 6, pp. 974-978, 1978.

[54] A. J. Bleyer, L. A. Donaldson, M. McIntosh, and P. L. Adams, "Relationship between underlying renal disease and renal transplantation outcome," American Journal of Kidney Diseases, vol. 37, no. 6, pp. 1152-1161, 2001.

[55] A. R. Nissenson and F. K. Port, "Outcome of end-stage renal disease in patients with rare causes of renal failure. III. Systemic/vascular disorders," Quarterly Journal of Medicine, vol. 74, no. 273, pp. 63-74, 1990.

[56] I. Batal, S. Bastacky, and P. Randhawa, "The outcome of kidney transplantation in patients with scleroderma," American Journal of Transplantation, vol. 7, no. 2, pp. 363-363, 2007, abstract 835.

[57] R. Shapiro, M. L. Jordan, V. P. Scantlebury et al., "A prospective, randomized trial of FK 506/prednisone vs FK 506/azathioprine/prednisone in renal transplant patients," Transplantation Proceedings, vol. 27, no. 1, pp. 814-817, 1995.

[58] W. Y. Cheung, I. W. Gibson, D. Rush, J. Jeffery, and M. Karpinski, "Late recurrence of scleroderma renal crisis in a renal transplant recipient despite angiotensin II blockade," American Journal of Kidney Diseases, vol. 45, no. 5, pp. 930934, 2005.

[59] P.-T. T. Pham, P.-C. T. Pham, G. M. Danovitch et al., "Predictors and risk factors for recurrent scleroderma renal crisis in the kidney allograft: case report and review of the literature," American Journal of Transplantation, vol. 5, no. 10, pp. 2565-2569, 2005.

[60] P. Liptak and B. Ivanyi, "Primer: histopathology of calcineurin-inhibitor toxicity in renal allografts," Nature Clinical Practice Nephrology, vol. 2, no. 7, pp. 398-404, 2006. 


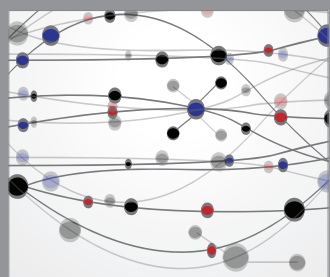

The Scientific World Journal
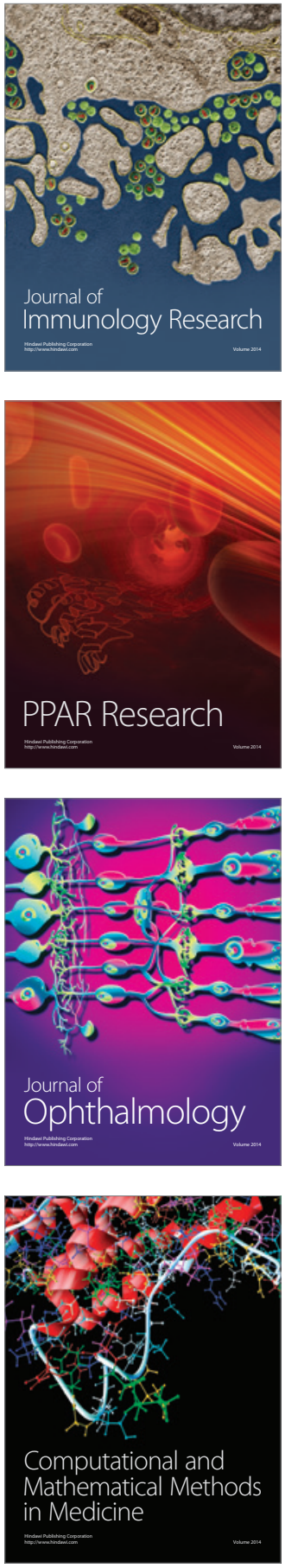

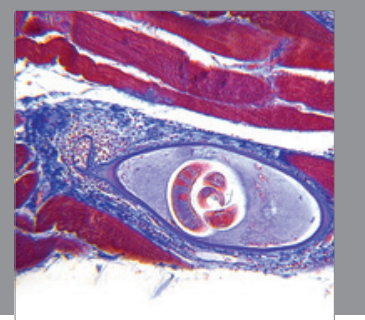

Gastroenterology

Research and Practice
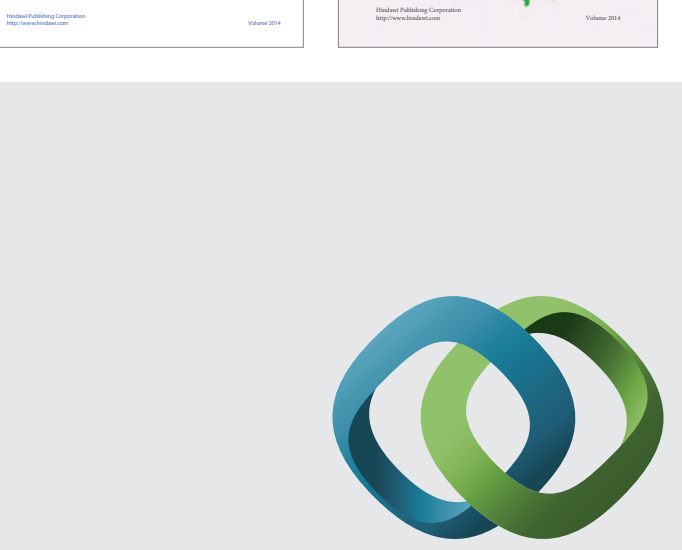

\section{Hindawi}

Submit your manuscripts at

http://www.hindawi.com
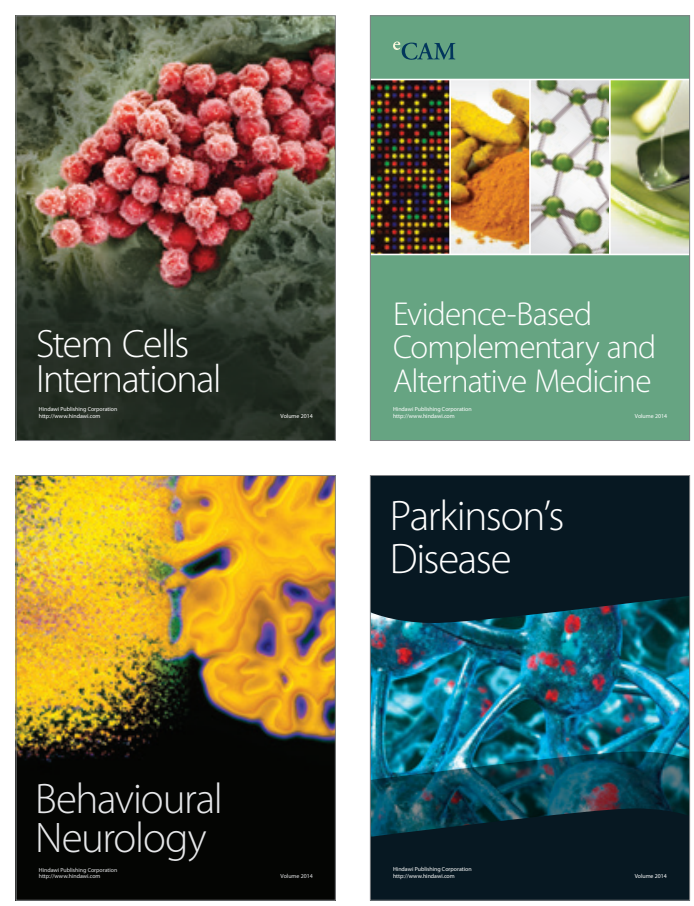

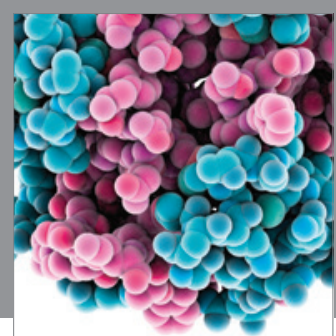

Journal of
Diabetes Research

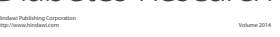

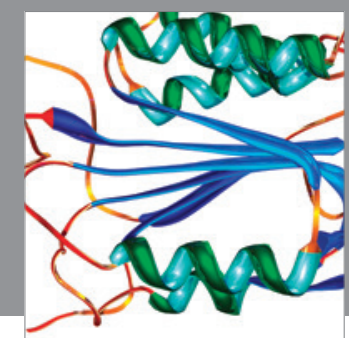

Disease Markers
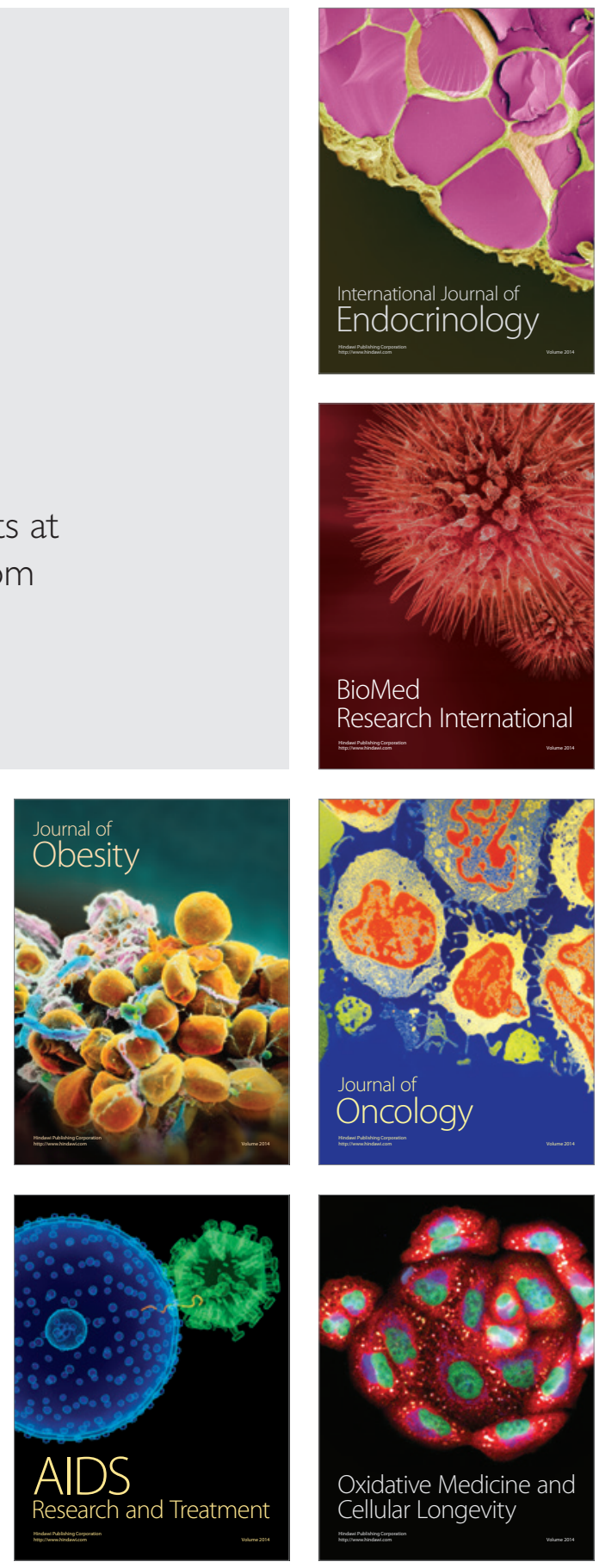